\title{
Measuring hindfoot alignment radiographically: the long axial view is more reliable than the hindfoot alignment view
}

\author{
Mikel L. Reilingh • Lijkele Beimers • \\ Gabriëlle J. M. Tuijthof • Sjoerd A. S. Stufkens • \\ Mario Maas • C. Niek van Dijk
}

Received: 14 September 2009 /Revised: 11 November 2009/Accepted: 3 December 2009 /Published online: 9 January 2010

(C) The Author(s) 2010. This article is published with open access at Springerlink.com

\begin{abstract}
Background Hindfoot malalignment is a recognized cause of foot and ankle disability. For preoperative planning and clinical follow-up, reliable radiographic assessment of hindfoot alignment is important. The long axial radiographic view and the hindfoot alignment view are commonly used for this purpose. However, their comparative reliabilities are unknown. As hindfoot varus or valgus malalignment is most pronounced during mid-stance of gait, a unilateral weightbearing stance, in comparison with a bilateral stance, could increase measurement reliability. The purpose of this study was to compare the intra- and interobserver reliability of hindfoot alignment measurements of both radiographic views in bilateral and unilateral stance.

Materials and methods A hindfoot alignment view and a long axial view were acquired from 18 healthy volunteers in bilateral and unilateral weight-bearing stances. Hindfoot alignment was defined as the angular deviation between the
\end{abstract}

M. L. Reilingh $\cdot$ L. Beimers $\cdot$ G. J. M. Tuijthof $(\bowtie) \cdot$

S. A. S. Stufkens $\cdot$ C. N. van Dijk

Department of Orthopaedic Surgery, Academic Medical Center,

Amsterdam, The Netherlands

e-mail: g.j.tuijthof@amc.uva.nl

M. L. Reilingh

e-mail: m.1.reilingh@amc.uva.nl

L. Beimers

e-mail: 1.beimers@amc.uva.nl

S. A. S. Stufkens

e-mail: stufkens@gmail.com

C. N. van Dijk

e-mail: m.lammerts@amc.uva.nl

\section{Maas}

Department of Radiology, Academic Medical Center,

Amsterdam, The Netherlands

e-mail:m.maas@amc.uva.nl tibial anatomical axis and the calcaneus longitudinal axis from the radiographs. Repeat measurements of hindfoot alignment were performed by nine orthopaedic examiners. Results Measurements from the hindfoot alignment view gave intra- and interclass correlation coefficients (CCs) of 0.72 and 0.58 , respectively, for bilateral stance and 0.91 and 0.49 , respectively, for unilateral stance. The long axial view showed, respectively, intra- and interclass CCs of 0.93 and 0.79 for bilateral stance and 0.91 and 0.58 for unilateral stance.

Conclusion The long axial view is more reliable than the hindfoot alignment view or the angular measurement of hindfoot alignment. Although intra-observer reliability is good/excellent for both methods, only the long axial view leads to good interobserver reliability. A unilateral weightbearing stance does not lead to greater reliability of measurement.

Keywords Intra-observer - Interobserver · Reliability · Hindfoot alignment view $\cdot$ Long axial view

\section{Introduction}

Hindfoot varus or valgus malalignment is a recognized cause of persisting foot and ankle disability and may result in degenerative joint disease [1]. For surgical correction of malalignment [2-10], the reliable preoperative determination of hindfoot alignment is important. Routine anteroposterior and lateral weight-bearing radiographs of the ankle are unsuitable, because of superimposition of the foot and ankle bones [11]. Two other radiographic projections have been described to overcome this problem: the hindfoot alignment view [11-14] (Fig. 1) and the long axial view [15-17] (Fig. 2). Measurements of hindfoot alignment on both views 
Fig. 1 a The hindfoot alignment view. The inclination angle of the beam is $20^{\circ}$ to the floor. The film cassette is perpendicular to the central beam of the radiation source. b A random example of a radiograph showing the hindfoot alignment view

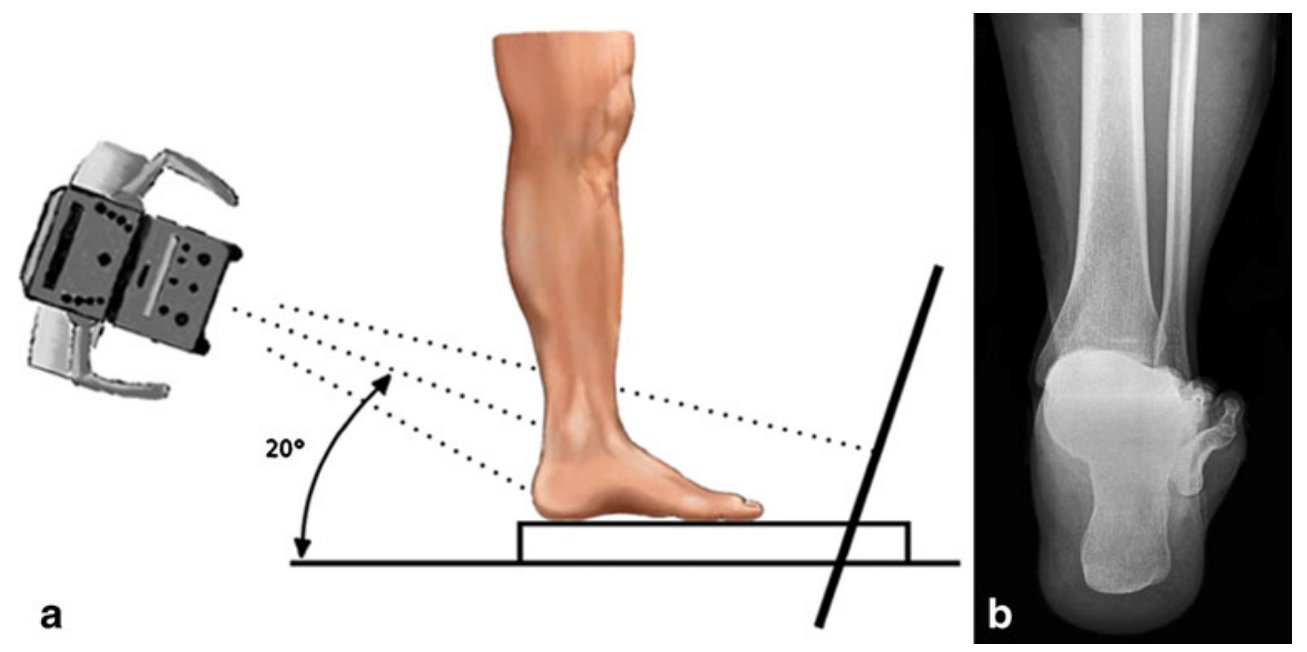

have been compared by Lamm et al. [15], who reported a significant correlation between both methods in a bilateral weight-bearing stance. However, the reliability of both views has not yet been compared.

We were also interested in determining whether a unilateral weight-bearing stance position when radiographs were being taken would increase the reliability. During the mid-stance phase of gait, there is a maximum eversion position of the hindfoot $[18,19]$, as the centre of gravity of the body will move to the lateral side of the weight-bearing foot when the subject is keeping balance. A maximum eversion position of the hindfoot will lock the talar and calcaneus bones in a fixed end position, which possibly leads to a larger angular deviation in the ankle joint [20]. Based on this, we expected that, in a unilateral weightbearing stance, varus or valgus hindfoot malalignment would potentially become more pronounced. A larger angle is generally easier to measure, which could increase the reliability of measurements.
In this study we addressed the question: are the intra- and interobserver reliabilities of measuring hindfoot alignment using the hindfoot alignment view and the long axial view significantly different? Additionally, we hypothesized that a unilateral weight-bearing stance would increase measurement reliability. Therefore, the two views were compared in bilateral and unilateral weight-bearing stance by one measuring method.

\section{Materials and methods}

\section{Population}

With approval from the ethics review committee, we recruited 18 healthy volunteers without ankle complaints. Our sample size $(n=18)$ was based on the method of Walter et al. [21]. There was a minimum intraclass correlation coefficient (ICC) of 0.7 and an expected ICC of 0.9 , with a
Fig. 2 a The long axial view. The film cassette is lying on the floor and the subject is standing on the film cassette. The inclination angle of the beam is $45^{\circ}$ to the floor. b A random example of a radiograph showing the long axial view

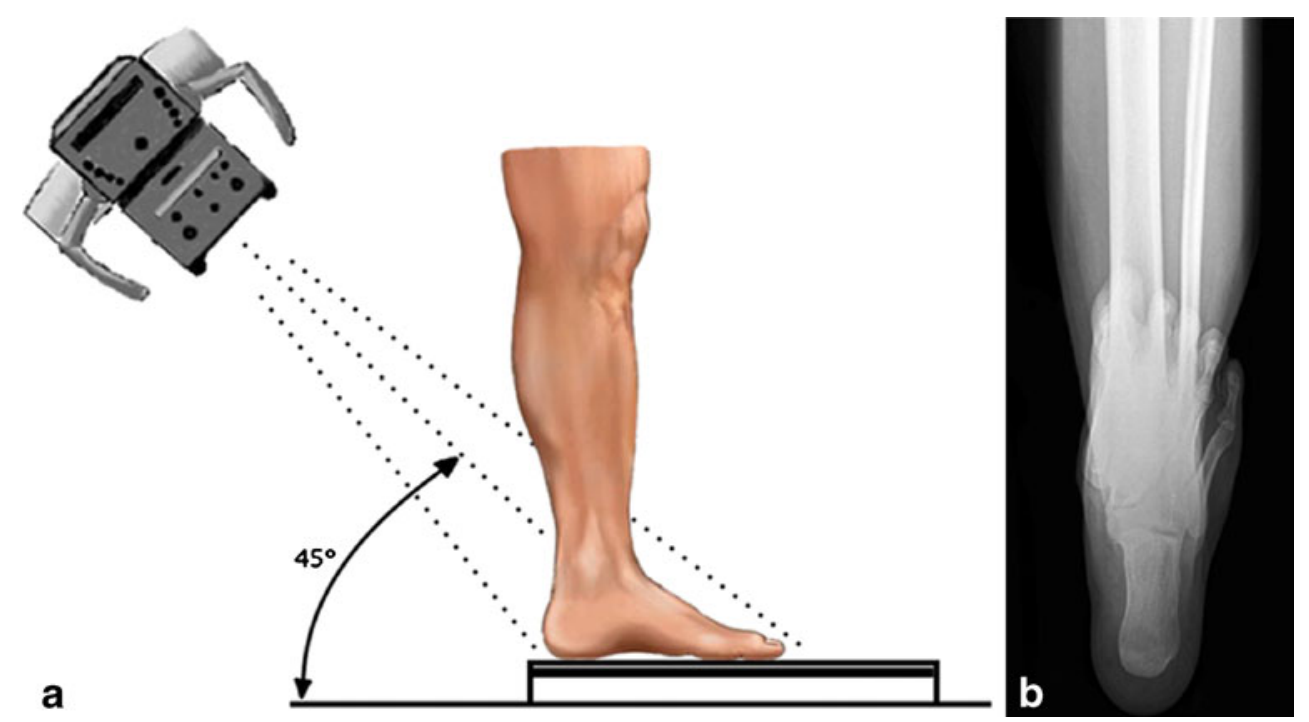


significance level of 0.05 and power of $80 \%$. The mean age was 29 years (range 17-52 years). There were six men and 12 women. Varus hindfoot alignment was seen in seven volunteers; eight volunteers had a valgus hindfoot alignment, and three had a neutral hindfoot alignment.

\section{Radiographic technique}

For the hindfoot alignment view, a cassette-holding box with a Plexiglas surface was used [12]. The settings for the radiation source was $4 \mathrm{mAs}$ and $50 \mathrm{kV}$, with a focus distance of $100 \mathrm{~cm}$, with the beam pointed at the ankle joint. The inclination angle of the beam was $20^{\circ}$ to the floor (Fig. 1). The film cassette was positioned perpendicular to the central beam of the radiation source.

To record the long axial view, the film cassette was lying flat on the floor [15]. Volunteers stood on the cassette and were asked to hold their ankle in $10^{\circ}$ of dorsal flexion, which was verified by a goniometer. The beam settings were the same as for the hindfoot alignment view. The inclination angle of the beam was $45^{\circ}$ to the floor (Fig. 2).

\section{Foot position}

The volunteers were placed in a predefined fixed foot position to minimize any influence on the measurements. Both feet were positioned by being internally rotated so that they were parallel. In the bilateral stance, the feet were positioned $80 \mathrm{~mm}$ apart, with the medial foot border parallel. From a biomechanical perspective, there was no significant difference in alignment between weight bearing in a natural stance of external rotation and a stance with both feet internally rotated [11]. After images of the subjects in a weight-bearing bilateral stance had been obtained, radiographs in the weight-bearing unilateral stance were made. The volunteers were asked to lift their unloaded left leg $50 \mathrm{~mm}$ up from the floor and a half foot length in front of the loaded leg.

\section{Measurement methods}

In the literature, the weight-bearing axis of the lower leg is routinely determined by the mid-diaphyseal axis of the tibia [11-16, 22, 23]. For the calcaneus, different methods exist to determine its axis in radiographic views. To compare both alignment views, we chose one measuring method, as described below.

In their study Saltzman and el-Khoury [12] measured the distance between the most distal part of the calcaneus and the longitudinal axis of the tibia. Their measurement method showed high reliability; however, this method only distinguished between three modes: neutral, valgus (the most distal part of the calcaneus was located lateral to the longitudinal axis of the tibia) and varus (the most distal part of the calcaneus was located medial to the longitudinal axis of the tibia). For the planning of corrective treatment, it is more desirable to determine a quantitative angle of malalignment. Therefore, an angular measure of the mid-diaphyseal line of the tibia to the mid-diaphyseal line of the calcaneus was chosen, which is also frequently suggested in the literature $[11,16,20,24]$. To determine the mid-diaphyseal line of the calcaneus, we applied the $40-60 \%$ division based on the description by Robinson et al. [25]. Using this radiographic measurement, we expected that the calcaneal mid-diaphyseal line could be determined more accurately. As this description was incomplete, a fixed definition was set for the levels at which the marks for the calcaneus axis had to be drawn. At a distance of $7 \mathrm{~mm}$ from the most distal part of the calcaneus, a horizontal line was drawn (Figs. 3 and 4). The width of the calcaneus at this level was divided into a 40\%:60\% ratio, where the length of $40 \%$ extended from the lateral side. A second line was drawn horizontally at $20 \mathrm{~mm}$ from the most distal part of the calcaneus in the hindfoot alignment view (Fig. 3) and $30 \mathrm{~mm}$ in the long axial view (Fig. 4). The width of the calcaneus at this second level was bisected equally. The

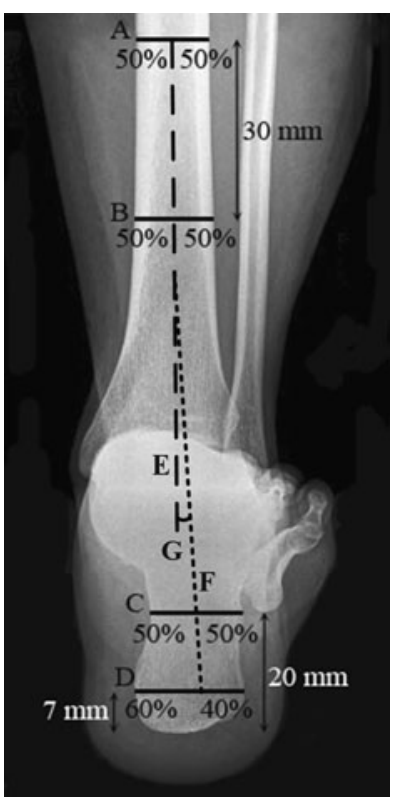

Fig. 3 Measurement method for the hindfoot alignment view. We defined the mid-diaphyseal axis of the tibia by bisecting the tibia into two mid-diaphyseal points (lines $A$ and B) $30 \mathrm{~mm}$ apart and extending the line distally (line $E$ ). The mid-diaphyseal axis of the calcaneus is defined by a line through two points in the calcaneus. At a distance of $7 \mathrm{~mm}$ from the most distal part of the calcaneus, a horizontal line is drawn (line D). Line D is divided into a $40 \%: 60 \%$ ratio, where the length of the $40 \%$ line is measured from the lateral side. A second line (line $C$ ) is drawn horizontally, $20 \mathrm{~mm}$ from the most distal part of the calcaneus. The calcaneus axis (line $F$ ) is drawn by connecting the $40 \%$ mark at line $\mathrm{D}$ and the bisected line $\mathrm{C}$. The hindfoot angle $(G)$ is the angle between lines $\mathrm{E}$ and $\mathrm{F}$ 


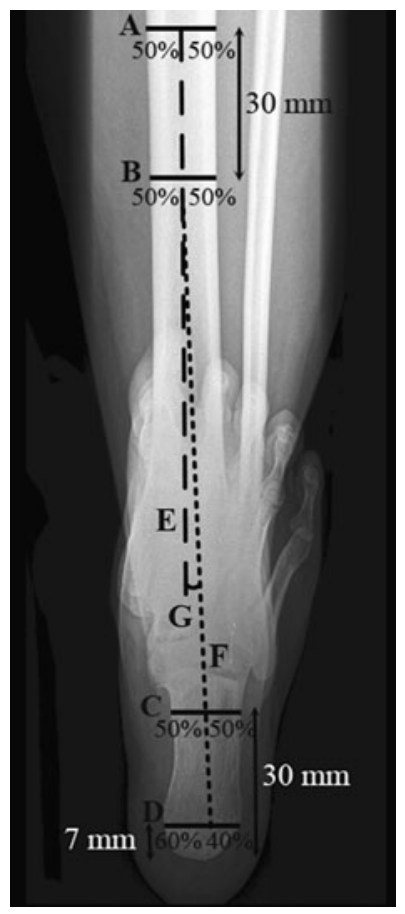

Fig. 4 Measurement method for the long axial view. We defined the mid-diaphyseal axis of the tibia by bisecting the tibia into two mid-diaphyseal points (lines $A$ and $B) 30 \mathrm{~mm}$ apart and extending the line distally (line $E$ ). The mid-diaphyseal axis of the calcaneus is defined by a line through two points in the calcaneus. At a distance of $7 \mathrm{~mm}$ from the most distal part of the calcaneus, a horizontal line is drawn (line $D$ ). Line D is divided into a $40 \%: 60 \%$ ratio, where the length of the $40 \%$ line is measured from the lateral side. A second line (line $C$ ) is drawn horizontally, $30 \mathrm{~mm}$ from the most distal part of the calcaneus. The calcaneus axis (line $F$ ) is drawn by connecting the $40 \%$ mark at line $\mathrm{D}$ and the bisected line $\mathrm{C}$. The hindfoot angle $(G)$ is the angle between lines $\mathrm{E}$ and $\mathrm{F}$

calcaneus axis was drawn by a line connecting the $40 \%$ mark at the first level and the bisecting mark at the second level.

In the images of both the hindfoot alignment view and the long axial view, the weight-bearing axis of the lower leg was determined by the mid-diaphyseal axis of the tibia. We defined this axis by bisecting the tibia into two mid-diaphyseal points $30 \mathrm{~mm}$ apart and extending the line distally (Figs. 3 and 4).

\section{Examiners}

Three experienced orthopaedic staff members and six orthopaedic residents measured the series of radiographs. For each volunteer, four radiographs of the right ankle were acquired and each radiograph was assessed three times; thus, a total of 216 angles had to be measured by each examiner. We randomly divided the images of the volunteers into three groups of equal size. The radiographs of each group of six volunteers were measured by three orthopaedic examiners of whom at least one was a senior staff member, thereby reducing the total number of measurements to 72 per examiner. In the third group the measurements were only performed two times to assess the intra-observer reliability.

\section{Statistics}

The mean angular differences and standard deviations (SDs) were calculated for repetitive measurements by individual examiners as well as between examiners.

To assess the reliability, we calculated the intraclass correlation coefficients [ICCs, type $(2, k)$, single measurer] with SPSS version 16.0 (SPSS Inc., Chicago, IL, USA). An ICC of 0.85 or higher indicates good reliability $(P<0.01)$ (26-28).

To determine an overall intra-observer reliability, we calculated the mean ICC of all three groups. To determine the interobserver reliability, we grouped the measurements of all volunteers, as it was irrelevant for this calculation that not all volunteers were measured by the same examiners.

\section{Results}

Measurements using the hindfoot alignment view gave respective intra- and interclass correlation coefficients of 0.72 and 0.58 for bilateral stance and 0.91 and 0.49 for unilateral weight-bearing stance. The long axial view showed respective intra- and inter correlation coefficients of 0.93 and 0.79 for bilateral stance and 0.91 and 0.58 for unilateral weight-bearing stance. For both methods, the intra-observer ICCs were good and fair for both bilateral stance and unilateral weight-bearing stance. The interobserver ICC was only good in the long axial view for bilateral stance (Tables 1 and 2).

The mean angular difference in the hindfoot alignment view was $2.2^{\circ}$ in bilateral stance and $1.8^{\circ}$ in unilateral weight-bearing stance for repeated measurements by the

Table 1 Intra-observer reliability of angular measurements of hindfoot alignment using two different radiographic views

\begin{tabular}{|c|c|c|c|c|}
\hline \multirow[t]{2}{*}{ Parameter } & \multicolumn{2}{|c|}{$\begin{array}{l}\text { Hindfoot alignment } \\
\text { view }\end{array}$} & \multicolumn{2}{|c|}{ Long axial view } \\
\hline & $\begin{array}{l}\text { Bilateral } \\
\text { stance }\end{array}$ & $\begin{array}{l}\text { Unilateral } \\
\text { stance }\end{array}$ & $\begin{array}{l}\text { Bilateral } \\
\text { stance }\end{array}$ & $\begin{array}{l}\text { Unilateral } \\
\text { stance }\end{array}$ \\
\hline $\begin{array}{l}\text { Mean of } \\
\text { differences (SD) }\end{array}$ & $\begin{array}{l}2.2^{\circ} \\
\left(1.7^{\circ}\right)\end{array}$ & $\begin{array}{l}1.8^{\circ} \\
\left(1.7^{\circ}\right)\end{array}$ & $\begin{array}{l}1.1^{\circ} \\
\left(1.1^{\circ}\right)\end{array}$ & $\begin{array}{l}1.4^{\circ} \\
\left(1.1^{\circ}\right)\end{array}$ \\
\hline $\begin{array}{l}\text { Mean of } \\
\quad \text { differences (SD) }\end{array}$ & $0.72 *$ & $0.91 *$ & $0.93 *$ & $0.91^{*}$ \\
\hline
\end{tabular}

$* P<0.01$ 
Table 2 Interobserver reliability for angular measurements of hindfoot alignment using two different radiographic views

\begin{tabular}{llllll}
\hline Parameter & \multicolumn{2}{l}{$\begin{array}{l}\text { Hindfoot alignment } \\
\text { view }\end{array}$} & & \multicolumn{2}{l}{ Long axial view } \\
\cline { 2 - 3 } \cline { 5 - 6 } \cline { 5 - 6 } & $\begin{array}{l}\text { Bilateral } \\
\text { stance }\end{array}$ & $\begin{array}{l}\text { Unilateral } \\
\text { stance }\end{array}$ & & $\begin{array}{l}\text { Bilateral } \\
\text { stance }\end{array}$ & $\begin{array}{l}\text { Unilateral } \\
\text { stance }\end{array}$ \\
\hline $\begin{array}{l}\text { Mean of } \\
\text { differences (SD) }\end{array}$ & $\begin{array}{l}3.3^{\circ} \\
\left(3.9^{\circ}\right)\end{array}$ & $\begin{array}{l}4.3^{\circ} \\
\left(3.8^{\circ}\right)\end{array}$ & & $\begin{array}{l}2.2^{\circ} \\
\left(2.1^{\circ}\right)\end{array}$ & $\begin{array}{l}3.2^{\circ} \\
\left(2.6^{\circ}\right)\end{array}$ \\
$\begin{array}{c}\text { Mean of } \\
\text { differences (SD) }\end{array}$ & 0.58 & 0.49 & & $0.79^{*}$ & 0.58 \\
\hline
\end{tabular}

$* P<0.01$

examiners. The long axial view gave a mean angular difference for repeated measurements by the examiners of $1.1^{\circ}$ in bilateral stance and $1.4^{\circ}$ in unilateral weight-bearing stance. Between observers, the mean angular difference in the hindfoot alignment view was $3.3^{\circ}$ in bilateral stance and $4.3^{\circ}$ in unilateral weight-bearing stance. The long axial view gave a mean angular difference between examiners of $2.2^{\circ}$ in bilateral stance and $3.2^{\circ}$ in unilateral weight-bearing stance (Tables 1 and 2).

\section{Discussion}

The results showed that the long axial hindfoot view had greater intra- and inter-rater reliability than the hindfoot alignment view in angular measurement of hindfoot alignment. The intra-observer reliability was, however, good to excellent for both methods. The interobserver reliability was only good for the long axial view (Tables 1 and 2). Furthermore, a unilateral weight-bearing stance did not lead to a greater measuring reliability for either radiographic view.

In 1995 Saltzman and el-Khoury reported an interobserver correlation coefficient of 0.97 for the hindfoot alignment view using only two examiners [12]. We found an interobserver correlation coefficient of 0.58 for the hindfoot alignment view. However, in our study, a different measurement method was used: an angular measurement of the mid-diaphyseal axis of the tibia and calcaneus, as suggested in the literature [11, $16,20,24]$. In our opinion, angular assessment should be performed to determine the level of the deformity, because this angle indicates the amount of surgical correction that would be required [16]. In a normal radiograph of an aligned hindfoot, the mid-diaphyseal axis of the calcaneus should be parallel $\left(0^{\circ}\right)$ to the mid-diaphyseal axis of the tibia, but its location is $5-10 \mathrm{~mm}$ lateral to the mid-diaphyseal axis of the tibia [16]. The end goal of surgical correction is anatomic realignment of the hindfoot, which is usually in a neutral position. Therefore, the preoperative hindfoot alignment should be determined as accurately as possible. A maximum difference of $1^{\circ}$ would be ideal [20]. In the long axial view, we found a mean angular intra-observer difference of $1.1^{\circ}$ and a mean angular interobserver difference of $2.2^{\circ}$ in bilateral stance. On the other hand, operative treatment is usually performed to correct more severe cases of hindfoot deformity, and, in that view the accuracy that was found is acceptable.

In our study the reliability of the hindfoot alignment view was less favourable than that of the long axial view. A possible explanation for the different results could be that, due to the inclination angle of the beam, the projected height of the calcaneus was shorter in the hindfoot alignment view than in the long axial view. The smaller the distance between two markers that define an axis, the more effect small measurement errors have on the orientation of that axis.

Although we performed a power analysis, the population of volunteers could have caused outliers that affected the results. Furthermore, we visually selected the volunteers for having a wide range of hindfoot alignment to imitate clinical practice as closely as possible; however, no volunteers had extreme ankle deformities. Therefore, the entire range of hindfoot deformities with extreme valgus or varus was not investigated. Additionally, not all examiners measured all images. The observers as well as the volunteers were divided into three groups of equal size. The radiographs of each group of six volunteers were measured by three orthopaedic examiners. However, the division of groups had no influence on the measurement of the interobserver reliability.

The hypothesis that the additional angular eversion rotation of the calcaneus opposed to the tibia during mid-stance of gait would give greater reliability was rejected, because no significant difference was found in the measurement of the hindfoot alignment in unilateral or bilateral weight-bearing stance. One reason could be that radiographic images were taken with the volunteers in a static position, as opposed to the dynamics of gait. Hamill et al. reported that static lower extremity measurements have limited value in predicting dynamic lower extremity function to any great degree [29]. Furthermore, the volunteers were allowed to keep their balance by using their hands and possibly partly corrected hindfoot valgus or varus.

Our conclusion was that the bilateral long axial view is more reliable than the hindfoot alignment view for measuring hindfoot alignment. Unilateral weight-bearing stance does not lead to greater measuring reliability of hindfoot alignment. For clinical and research purposes, it is recommended that the long axial hindfoot view in bilateral stance be used for radiographic assessment of hindfoot alignment. 
Open Access This article is distributed under the terms of the Creative Commons Attribution Noncommercial License which permits any noncommercial use, distribution, and reproduction in any medium, provided the original author(s) and source are credited.

\section{References}

1. Hayashi K, Tanaka Y, Kumai T, Sugimoto K, Takakura Y. Correlation of compensatory alignment of the subtalar joint to the progression of primary osteoarthritis of the ankle. Foot Ankle Int. 2008;29:400-6.

2. Takakura Y, Takaoka T, Tanaka Y, Yajima H, Tamai S. Results of opening-wedge osteotomy for the treatment of a post-traumatic varus deformity of the ankle. J Bone Jt Surg Am. 1998;80:213-18.

3. Hintermann B, Knupp M, Barg A. Osteotomies of the distal tibia and hindfoot for ankle realignment. Orthopade. 2008;37:212-13.

4. Stamatis ED, Myerson MS. Supramalleolar osteotomy: indications and technique. Foot Ankle Clin. 2003;8:317-33.

5. Tanaka Y, Takakura Y, Hayashi K, Taniguchi A, Kumai T, Sugimoto K. Low tibial osteotomy for varus-type osteoarthritis of the ankle. J Bone Joint Surg Br. 2006;88:909-13.

6. Stamatis ED, Cooper PS, Myerson MS. Supramalleolar osteotomy for the treatment of distal tibial angular deformities and arthritis of the ankle joint. Foot Ankle Int. 2003;24:754-64.

7. Sen C, Kocaoglu M, Eralp L, Cinar M. Correction of ankle and hindfoot deformities by supramalleolar osteotomy. Foot Ankle Int. 2003;24:22-8.

8. Trnka HJ, Easley ME, Myerson MS. The role of calcaneal osteotomies for correction of adult flatfoot. Clin Orthop. 1999;365:50-64.

9. Dierauer S, Schafer D, Hefti F. Osteotomies of the mid- and back-foot in recurrent club foot. Orthopade. 1999;28:117-24.

10. Catanzariti AR, Mendicino RW, King GL, Neerings B. Double calcaneal osteotomy: realignment considerations in eight patients. J Am Podiatr Med Assoc. 2005;95:53-9.

11. Johnson JE, Lamdan R, Granberry WF, Harris GF, Carrera GF. Hindfoot coronal alignment: a modified radiographic method. Foot Ankle Int. 1999;20:818-25.

12. Saltzman CL, el-Khoury GY. The hindfoot alignment view. Foot Ankle Int. 1995;16:572-6.

13. Cobey JC. Posterior roentgenogram of the foot. Clin Orthop. 1976;118:202-7.
14. Buck P, Morrey BF, Chao EY. The optimum position of arthrodesis of the ankle. A gait study of the knee and ankle. J Bone Joint Surg Am. 1987;69:1052-62.

15. Lamm BM, Mendicino RW, Catanzariti AR, Hillstrom HJ. Static rearfoot alignment: a comparison of clinical and radiographic measures. J Am Podiatr Med Assoc. 2005;95:26-33.

16. Mendicino RW, Catanzariti AR, Reeves CL, King GL. A systematic approach to evaluation of the rearfoot, ankle, and leg in reconstructive surgery. J Am Podiatr Med Assoc. 2005;95:2-12.

17. Kleiger B, Mankin HJ. A roentgenographic study of development of the calcaneus by means of the posterior tangential view. J Bone Joint Surg Am. 1961;43:961-9.

18. Torburn L, Perry J, Gronley JK. Assessment of rearfoot motion: passive positioning, one-legged standing, gait. Foot Ankle Int. 1998;19:688-93.

19. Pierrynowski MR, Smith SB. Rear foot inversion/eversion during gait relative to the subtalar joint neutral position. Foot Ankle Int. 1996; 17:406-12.

20. Smith-Oricchio K, Harris BA. Interrater reliability of subtalar neutral, calcaneal inversion and eversion. J Orthop Sports Phys Ther. 1990;12:10-5.

21. Walter SD, Eliasziw M, Donner A. Sample size and optimal designs for reliability studies. Stat Med. 1998;17:101-10.

22. Mendicino RW, Catanzariti AR, John S, Child B, Lamm BM. Long leg calcaneal axial and hindfoot alignment radiographic views for frontal plane assessment. J Am Podiatr Med Assoc. 2008;98:75-8.

23. Strash WW, Berardo P. Radiographic assessment of the hindfoot and ankle. Clin Podiatr Med Surg. 2004;21:295-304. v.

24. Tuijthof GJ, Herder JL, Scholten PE, van Dijk CN, Pistecky PV. Measuring alignment of the hindfoot. J Biomech Eng. 2004;126:357-62.

25. Robinson I, Dyson R, Halson-Brown S. Reliability of clinical and radiographic measurement or rearfoot alignment in a patient population. The Foot. 2001;11:238-48.

26. Muller R, Buttner P. A critical discussion of intraclass correlation coefficients. Stat Med. 1994;13:2465-76.

27. Deyo RA, Diehr P, Patrick DL. Reproducibility and responsiveness of health status measures. Statistics and strategies for evaluation. Control Clin Trials. 1991;12(4 Suppl):142S-58.

28. Sell KE, Verity TM, Worrell TW, Pease BJ, Wigglesworth J. Two measurement techniques for assessing subtalar joint position: a reliability study. J Orthop Sports Phys Ther. 1994;19:162-7.

29. Hamill J, Bates BT, Knutzen KM, Kirkpatrick GM. Relationship between selected static and dynamic lower extremity measures. Clin Biomech. 1989;4:217-25. 